\title{
Belegt der Fund einer „Schieferkohle“ die spätglaziale Vergletscherung der Immenstädter Nagelfluhberge?
}

\author{
Ralf Irmler \& Herbert Scholz*)
}

Zusammenfassung: Im Rahmen einer Diplomkartierung wurde im Gebiet der Immenstädter Nagelfluhberge (Faltenmolasse) am Sedererbach, einem südlichen Nebenbach der Weißach, auf ca. $1150 \mathrm{~m}$ Höhe zwischen Sedererstuiben und Buralpkopf, ein bislang unbekanntes Vorkommen einer quartären „Schieferkohle“ entdeckt. Es handelt sich um mehrere geringmächtige Einschaltungen aus organischem Material in sehr dicht gelagerte Schluffe und Sande, die von 2 bis max. $4 \mathrm{~m}$ mächtigen sterilen Kiesen überlagert werden. Innerhalb des gleichfalls sehr festen, torfartigen Materials ließen sich kaum Hölzer, dafür aber vor allem Reste von Sphagnaceen (Torfmoosen) und Diatomeen nachweisen.

Aufgrund der Morphologie und der Geländebefunde ist es unwahrscheinlich, dass die augenscheinliche Überkonsolidierung des Materials auf eine ehemals mächtigere Überdeckung durch inzwischen abgetragene Sedimente oder Rutschmassen zurückzuführen ist. Angesichts der Position der Fundstelle direkt unterhalb eines großen Kares auf der Nordseite der über 1800 m hohen Rindalphorn-Hochgrat-Kette ist sie wohl eher mit einer Überfahrung durch einen Lokalgletscher erklärbar. Im Rahmen eines Kompressionsversuches mit behinderter Seitenausdehnung, der an diesem Material durchgeführt wurde, ließ sich durch Bestimmung des Casagrande-Kni-

* Anschrift der Verfasser: Dipl.-Geol. Dr. Ralf IrmLER, Institut für Geographie der Friedrich-SchillerUniversität Jena, Löbdergraben 32, D-07743 Jena, Dipl.-Geol. Dr. Herbert Scholz, Privatdozent am Lehrstuhl für Allgemeine, Angewandte und Ingenieur-Geologie der Technischen Universität München, Arcisstr. 21, D-80290 München ckes eine mögliche, ehemalige Eisüberlagerung von gut $20 \mathrm{~m}$ abschätzen.

Drei ${ }^{14} \mathrm{C}$-Datierungen ergaben mit $11930 \pm 80$, $11950 \pm 60$ und $11620 \pm 80$ Jahren BP überraschend junge Sedimentationsalter der Proben, die damit offenbar aus dem älteren Teil des Alleröd-Interstadials stammen. Als Zeitpunkt für eine mögliche Eisüberfahrung bleibt demnach nur die Jüngere Dryaszeit übrig (Egesen-Stadium), für die an dieser Stelle eine überraschend kräftige Depression der "Schneegrenze“ von mindestens $450 \mathrm{~m}$ gegenüber einer heutigen hypothetischen „Schneegrenze“ angenommen werden muss. Es ist wohl davon auszugehen, dass die großen Kare im Gebiet der Immenstädter Nagelfluhkette das letzte Mal kurz vor Beginn des Holozäns vergletschert waren.

Abstract: During field work a so long unknown occurence of a quaternary lignite has been discovered within the valley of Sedererbach, a southern tributary of River Weißach, situated in the conglomeratic mountains chains (folded Molasse) near Immenstadt in southwestern Bavaria (Allgäu), just below the mountains of Sedererstuiben and Buralpkopf in an altitude of $1150 \mathrm{~m}$. The occurence consists of several thin organic intercalations within strongly consolidated silts and sands, covered with 2 up to a maximum of $4 \mathrm{~m}$ of gravel lacking any organic material. Although woody fragments are rare within the peat-like material, which is strongly consolidated as well, many remains of mosses (Sphagnaceans) and Diatoms can be detected.

The obvious overconsolidation of the material can not be interpreted as the result of the load of a formerly thicker sedimentary cover which may have been eroded in the meantime. The situation 
of the occurrence just below of a wide cirque on the northern side of the more than $1800 \mathrm{~m}$ high Rindalphorn-Hochgrat mountain chain stresses the obvious possibility of a local glacier that could have overridden the site. Due to compression experiments with samples of this material and the determination of the Casagrande-Point showed, that an ice-cover of about $22 \mathrm{~m}$ is most likely.

Three ${ }^{14} \mathrm{C}$ - datings showed surprisingly young sedimentation ages of the samples of $11930 \pm 80$, $11950 \pm 60$ und $11620 \pm 80 \mathrm{yBP}$, which seem to derive form the Alleröd Interstadial. Therefore the glacier advance could only have happened during the subsequent climatic depression of Younger Dryas (Egesen Stage), which leads to the assumption of a surprisingly strong snow-line depression of at least $450 \mathrm{~m}$. These data are the first to give strong evidence for a last glaciation of the cirques within the mountains chains near Immenstadt in southwestern Bavaria just before the end of Pleistocene.

\section{Geographischer und geologischer Rahmen}

Im Rahmen einer Diplomkartierung im oberen Weißachtal (Irmler 2001) südwestlich von Immenstadt im Allgäu (Abb. 1) wurde im Tal des Sederbaches ein bislang unbekanntes Vorkommen einer quartären "Schieferkohle“ entdeckt. Die Fundstelle (R: 35.60248, H: 52.66244) liegt unmittelbar am Sedererbach in einer Höhe von etwa $1150 \mathrm{~m}$. Der Bach entspringt im Sedererkar, das an der Nordflanke der Rindalphorn-Hochgrat-Kette zwischen $\mathrm{Bu}$ ralpkopf (1772 m) und Stuiben $(1749 \mathrm{~m})$ bzw. dessen Nebengipfel Sedererstuiben $(1737 \mathrm{~m})$ im Gebiet der Immenstädter Nagelfluhberge liegt, und mündet im weiten EhrenschwangBecken in die Weißach. In westlicher Verlängerung des Gipfelgrates liegt der Hochgrat,

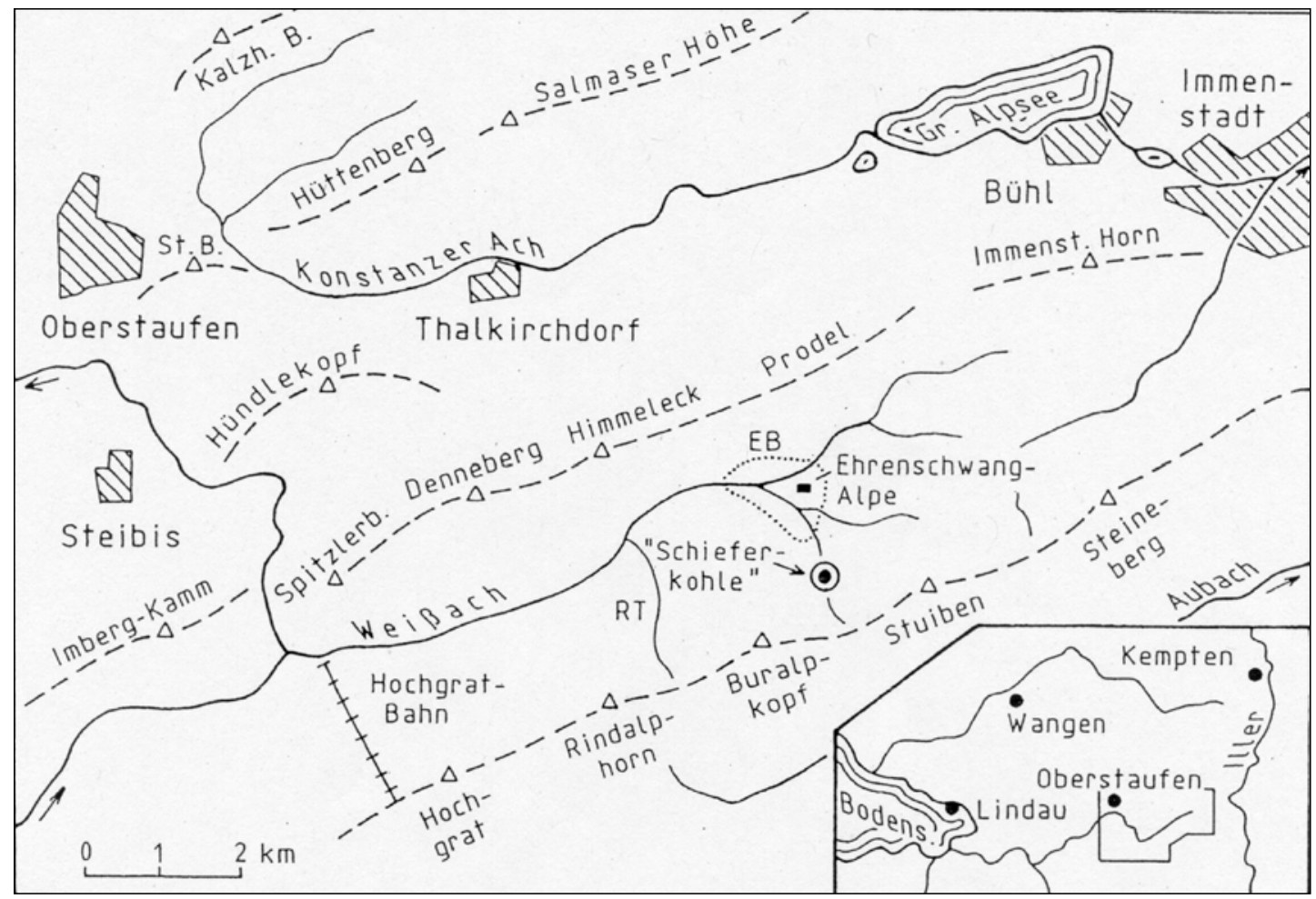

Abb. 1: Topographische Übersichtskarte der Immenstädter Nagelfluhberge. Eingezeichnet sind das etwas vereinfachte Gewässernetz, die wichtigsten Bergkämme und Gipfel sowie die Lage der „Schieferkohle“ am Sedererbach. EB = Ehrenschwang-Becken $\quad$ RT $=$ Rindalper Tobel 
der mit $1832 \mathrm{~m}$ die höchste Erhebung der Immenstädter Nagelfluhkette darstellt. Zwischen dieser und der deutlich niedrigeren Kette des Prodel im N ist das Weißachtal eingesenkt, das von der Weißach in Richtung W entwässert wird (Abb. 1).

Das untersuchte Gebiet liegt in der Allgäuer Faltenmolasse im Bereich der südlichsten Molassemulden (Hornschuppe und Steinebergmulde). Die WSW-ENE-streichenden Höhenrücken werden im Wesentlichen von Gesteinen der Unteren Süßwassermolasse aufgebaut, die man hier klassisch in die Weißach-, Steigbach- und Kojenschichten gliedert. Die Typlokalitäten dieser stratigraphischen Einheiten befinden sich alle in der näheren Umgebung des Weißachtales (Vollmayr 1958, Schwerd et al. 1983). Es handelt sich um Wechselfolgen von Konglomeraten, Ton-Schluff-Steinen und Sandsteinen. Alle hier aufgeschlossenen Molassegesteine sind steilgestellt und fallen mit 45 bis $70^{\circ}$ meist nach $S$ ein.

Tiefe Karmulden und deutlich ausgebildete, diese Kare umkränzende Wallsysteme, blockreiche glazigene Ablagerungen sowie konglomeratische Findlingsblöcke sind an der Nordflanke der Rindalphorn-Hochgrat-Kette weit verbreitet und deuten auf die Existenz zahlreicher kleiner kaltzeitlicher Lokalgletscher hin. Blockreiche glazigene Ablagerungen des gleichen Typs, die ausschließlich Lokalmaterial aus dem Hochgratgebiet enthalten, lassen sich stellenweise bis in die Umgebung von Steibis nachweisen. Daher ist anzunehmen, dass sich diese Lokalgletscher zeitweise zu einem Talgletscher im Weißachtal vereinigt haben müssen, der über die Engstelle von Imberg und Spitzlerberg hinaus bis in die Gegend des Weißachknies bei Oberstaufen vorgestoßen ist (Abb. 1). Im Weißachtal selbst sind deutliche morphologische Hinweise auf eine Talvergletscherung jedoch auf das obere Weißachtal zwischen Ehrenschwangalpe und Einmündung des Rindalper Tobels in die Weißach beschränkt.
Hier finden sich in einigen Tobeln und im Ehrenschwang-Becken zudem Aufschlüsse frischer glazigener Bildungen (IRMLER 2001), während weiter unten im Weißachtal alle eindeutig glazigenen Ablagerungen tiefgründig verwittert und entkalkt sind (Rogozinski 1998).

Die blockreichen glazigenen Ablagerungen, die ausschließlich Lokalmaterial aus dem Hochgratgebiet enthalten und durch ihren Gehalt an metergroßen Konglomeratblöcken sehr auffällig erscheinen, sind in der Umgebung von Steibis immer von jüngeren Fernmoränen überdeckt (Rogozinski 1998, Wimmer 1998). Somit ist davon auszugehen, dass nur in älteren Eiszeiten ein Talgletscher existierte, der das Weißachtal abwärts über die Engstelle von Imberg und Denneberg hinaus nach $\mathrm{N}$ vorstoßen konnte. In der Gegend des Weißachknies dürfte er sich zeitweise mit der Weißachzunge, einem Ast des Rhein-Bodensee-Vorlandgletschers vereinigt haben. Im Würm-Hochglazial hingegen scheinen nach Rogozinski (I 998) und IRMLER (200I) die meisten Kargletscher gerade einmal den Grund des Weißachtales erreicht zu haben (Abb. 2). Jedenfalls war die Vergletscherung des oberen Weißachtales in der letzten Eiszeit offenbar bedeutend geringer als bisher angenommen worden war (z.B. JÄCKLI 1970, WEINHARDT 1973, Scholz I995).

\section{Die „Schieferkohle“}

Der Aufschluss der "Schieferkohle“ befindet sich zu Füßen der Sedererwände, unmittelbar unterhalb eines kleinen Wasserfalles im Sedererbach in einer Höhe von etwa $1150 \mathrm{~m}$. Beiderseits des hier N-S-verlaufenden Baches sind an den Prallhängen horizontal lagernde quartäre Sedimente (Kiese und Schluffe) angeschnitten, die Horizonte mit organischem Material enthalten (Abb. 3). Die Basis des westlichen Aufschlusses bilden grau-braune Schluffe mit 


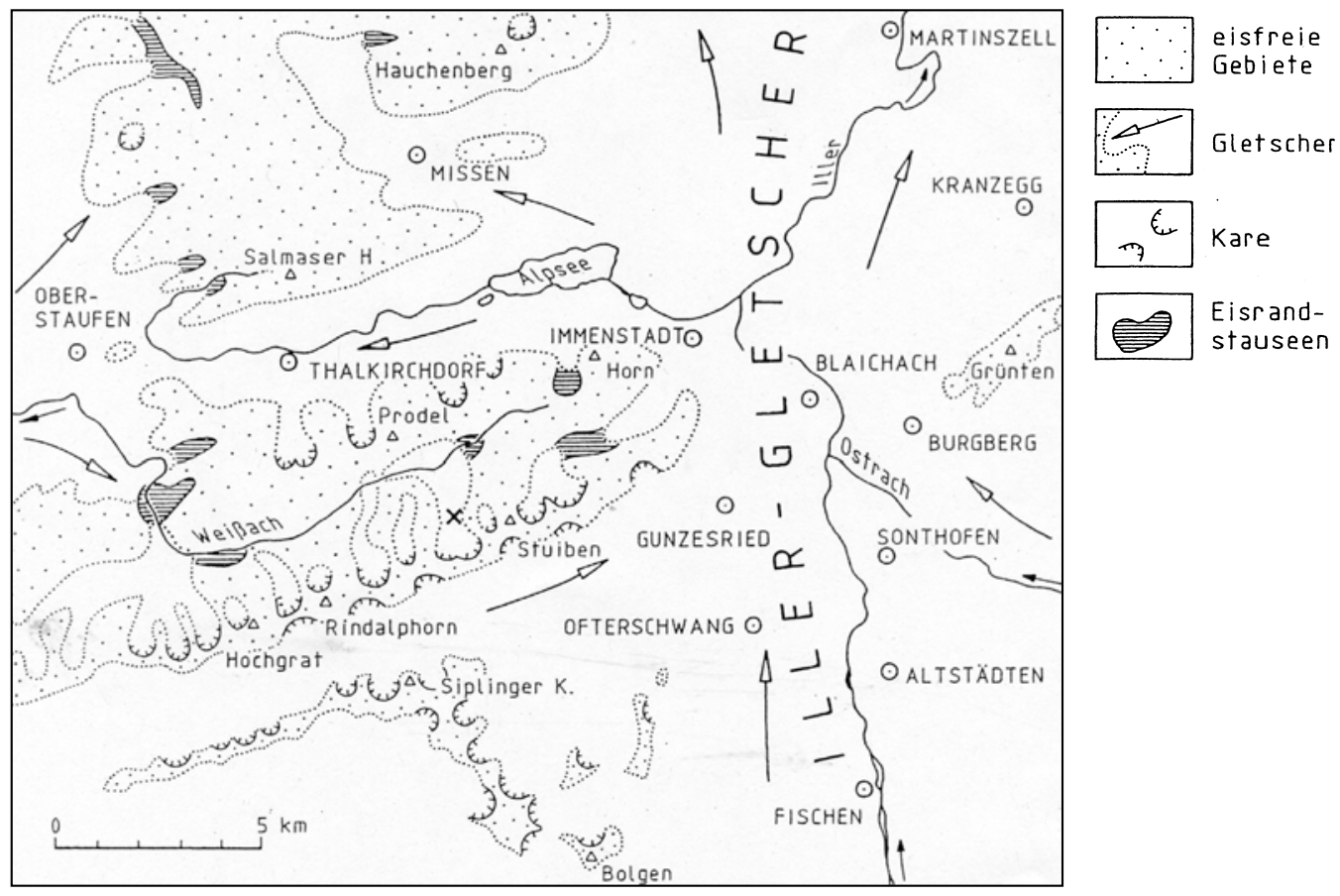

Abb. 2: Die Immenstädter Nagelfluhberge während des Vereisungsmaximums der Würmeiszeit (LGM). Rekonstruktionsversuch auf der Grundlage von geologischen Detailkartierungen von BüHRING (I993), Wimmer (1998), Rogozinski (i998), Irmler (200I) und Past (200i). Die Vergletscherung des oberen Weißachtales war in der letzten Eiszeit offenbar bedeutend geringer als bisher angenommen (vergl. z.B. Jäckli 1970, Weinhardt 1973 oder Scholz i995). Mit dem Kreuz ist die Lage der Schieferkohle im Sedererbach markiert.

stark angelösten, eckigen Komponenten und Gesteinssplittern. Wahrscheinlich handelt es sich um solifluidal umgelagerte, mit Steinen durchmischte Molassemergel. Dieses Material wird von einer etwa $60 \mathrm{~cm}$ mächtigen Folge von mehr oder weniger schluffreichen Kiesen und Sanden sowie reinen Schluffen überlagert, in die mehrere, maximal $4 \mathrm{~cm}$ dicke Horizonte aus organischem Material eingeschaltet sind. Im Hangenden der obersten dieser organischen Lagen folgen bis zur Geländeoberkante $2 \mathrm{~m}$ verstürztes, kiesiges Material, bei dem es sich um die gleichen, schlecht geschichteten Kiese handeln dürfte, die auch auf der anderen Bachseite aufgeschlossen sind.

Am östlichen Prallhang sind in einer ähnlichen Schichtfolge insgesamt nur drei Horizonte mit organischem Material eingeschaltet. Im Hangenden der obersten dieser Lagen folgen hier bis max. $4 \mathrm{~m}$ mächtige, sandige, schlecht geschichtete Kiese, in denen kein organisches Material gefunden werden konnte (Abb. 3). Die Gerölle in diesem Kies zeigen deutliche Imbrikation. Hinsichtlich ihrer Farbe und ihrer Sterilität erinnern sie eher an kaltzeitliche Schmelzwasserkiese als an warmzeitliche Bachsedimente. Bei allen Komponenten, die in dieser Schichtfolge vorkommen, scheint es sich um Fragmente von Sandsteinbänken oder Geröllen aus entfestigten Konglomeraten zu handeln, also um umgelagerte Molassegesteine. Die Horizonte mit organischem Material, aber auch alle anderen Sedimente im Liegenden der Kiese, machen einen ungewöhnlich festen, augenscheinlich 

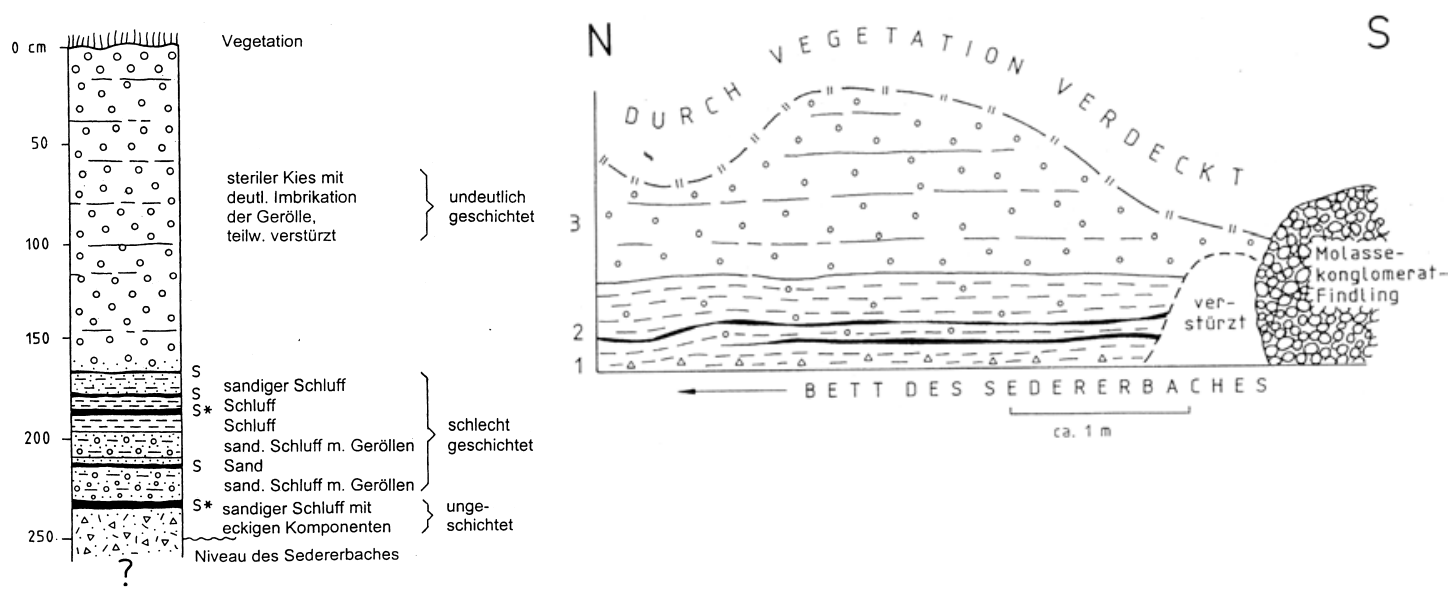

Abb. 3: Das „Schieferkohle“-Profil im Sedererbach.

Rechts: Schematische Übersichtsskizze vom östlichen Prallhang des Sedererbaches.

$3=$ undeutlich geschichteter, steriler Kies

2 = schlecht geschichtete Schluffe und Sande mit Geröllen und organischen Einschaltungen

1 = sandiger Schluff mit eckigen Komponenten, ungeschichtet

Links: Detailliertes Säulenprofil des westlichen Prallhanges im Sedererbach. Horizonte, die aus torfartigem, organischen Material bestehen, sind mit „S“ gekennzeichnet. Stellen, an denen Proben für die radiometrische Altersbestimmung entnommen worden sind, wurden zusätzlich mit einem Sternchen markiert.

überkonsolidierten Eindruck.

Die Lagen mit organischem Material sind braun gefärbt, faserig strukturiert und bestehen im wesentlichen aus Pflanzenresten. Schon mit bloßem Auge sind viele dünne, plattgedrückte Stengel erkennbar. Dickere Ästchen oder größere Holzreste fehlen völlig. Bei einer Untersuchung der Pflanzenreste unter der Stereolupe und dem Durchlichtmikroskop konnten vor allem zahlreiche Reste von Torfmoosen (Sphagnaceen) identifiziert werden. Daneben wurden schlecht erhaltene fädige Grünalgen und Diatomeen gefunden. Der Erhaltungszustand der ursprünglich holzigen Stengel war zu schlecht, um das Material näher bestimmen zu können. Die „Schieferkohle“ wurde von Frau Dr. H. Schneider am Geographischen Institut der Universität Jena pollenanalytisch untersucht. Die Ergebnisse belegen im Profilabschnitt 189 bis $235 \mathrm{~cm}$ (Abb. 3 links) eine einheitliche Entwicklung der regionalen Vegetation. Dabei fallen vor allem sehr hohe prozentuale Werte von Pinus, zugleich aber auch regelmäßige Nachweise von lichtliebenden Arten wie Juniperus, Cichorioidae, Chenopodiaceae, Artemisia und Poaceae auf. Anhand des Artenspektrums lässt sich ein ausgedehnter Kiefernwald im weiteren Umfeld der untersuchten Ablagerungen rekonstruieren, der an exponierten und lichten Standorten vor allem Raum für Offenlandgesellschaften bot. Im Bereich der Ablagerungen selbst entwickelte sich im untersuchten Zeitraum ein Moor, das zunächst vor allem durch vereinzelte Hochstauden, Farne, Sauergräser und vor allem Moosfarn (Selaginella) geprägt war. Im weiteren Verlauf charakterisierten jedoch in erster Linie Sauergräser, aber auch Wasserpflanzen (Callitriche-type) das Bild der lokalen Vegetation und lassen auf zunehmende Versumpfung schließen. Der Nachweis von Pollenkörnern der Gattungen Alnus und Corylus kann hier sicher als Beleg für anhaltende Umlagerungsprozesse angesehen werden.

Die mineralischen Einschaltungen zwischen 
den dünnen Torfhorizonten waren extrem arm an Palynomorphen. Die wenigen erhaltenen Pollenkörner waren stark korrodiert und ermöglichten deshalb keine Auswertung. Möglicherweise sind sie in einem sehr kurzen Zeitraum abgelagert worden. Zur pollenanalytischen Altersbestimmung vergl. Abschnitt 2.2.

\subsection{Kompressionsversuch an einer Probe}

\section{aus der „Schieferkohle“}

Um den subjektiven Eindruck der Konsolidierung der "Schieferkohle“ zu verifizieren, wurde der Grad der Verfestigung im Rahmen eines Kompressionsversuches bei behinderter Seitenausdehnung (DIN 18135) gemessen. Zur Bestimmung des Setzungsverhaltens wurde eine ungestörte Probe (Güteklasse 1 nach DIN 4021) mit einem Stechring $(71,4 \mathrm{~mm} \varnothing)$ gewonnen und im Kompressionsapparat stufenweise be-, ent- und wiederbelastet. Bei einem natürlichen Wassergehalt von 60 Gewichts-\% (bezogen auf Trockenmasse) ergab sich eine maximale Setzung von knapp $40 \%$.

Auffällig ist der konvexe Verlauf der halblogarithmischen Last-Zusammendrückungs-Kurve bei der Erstbelastung (Abb. 4 oben). Das heißt, die Kurve besitzt bei mäßiger Zunahme der Normalspannung eine flach gekrümmte Form, bei höheren Normalspannungen dagegen eine deutlich stärkere Neigung. Die Ursache hierfür wird im Überschreiten einer früheren Vorbelastung gesehen. Der Wendepunkt der Kurve wird als „Casagrande-Knick“ bezeichnet (Abb. 4 oben). Nach Prinz (1997: 34), Bölling (1971: 43) sowie der DIN 18135 lässt sich aus der Lage dieses Knickpunktes die ungefähre Größe der Vorbelastung bestimmen, die im vorliegenden Falle im Bereich von ca. $200 \mathrm{kN} / \mathrm{m}^{2}$ liegt.

Für diese augenscheinliche Überkonsolidierung der "Schieferkohle“ und der sie umgebenden schluffigen Sedimente ist eine Überlagerung durch 2 bis maximal $4 \mathrm{~m}$ Kies, wie sie derzeitig im Gelände vorzufinden ist (Abb. 3), nicht ausreichend. Keine Geländebefunde sprechen für eine ursprünglich deutlich höhere Überlagerung, etwa durch wesentlich mächtigere Bachsedimente oder durch Rutschmassen, die inzwischen der Erosion zum Opfer gefallen sein könnten. Eine für die Kompaktion ausreichend mächtige Überlagerung aus Schnee- und Lawinenmassen wäre aufgrund der Lage des Schieferkohlevorkommens denkbar. Allerdings ist der nötige Belastungszeitraum für eine derartige Kompaktion wohl als deutlich zu gering zu betrachten. Ein möglicher Grund für die hohe Vorbelastung angesichts der Lage der "Schieferkohle" könnte in einer Eisüberfahrung zu finden sein. Bei einer Interpretation der Ergebnisse des Kompressionsversuches (Abb. 4) in diesem Sinne würde sich bei einer angenommenen Eisdichte von $0,9 \mathrm{~g} / \mathrm{cm}^{3}$ eine Eisüberlagerung von gut $20 \mathrm{~m}$ ergeben.

$\mathrm{Zu}$ Vergleichszwecken wurde auch das Kompressionsverhalten eines holozänen, nicht vorbelasteten jungen Torfes aus einem Hochmoor östlich von München getestet. Die Torfprobe wurde aus einem etwa $700 \mathrm{~m}$ nördlich von Fürmoosen bei Kirchseeon (Landkreis Ebersberg) gelegenen Torfstich aus einer Tiefe von etwa $2 \mathrm{~m}$ entnommen. Die ursprüngliche Überlagerung durch Sedimente ist hier damit ähnlich hoch wie bei den „Schieferkohlen“ im Sedererbach. Es ergab sich ein zehnfach höherer natürlicher Wassergehalt (600 Gewichts-\%! bezogen auf die Trockenmasse), eine deutlich größere Zusammendrückbarkeit (85\%) und eine völlig anders gekrümmte, konkave Erstbelastungskurve (Abb. 4 unten).

Der Versuch, aus schluffigen Sedimenten des Schieferkohle-Profils gleichfalls Proben für Kompressionsversuche zu gewinnen, schlug leider fehl. Ein Teil des Materials erwies sich als sekundär stark aufgeweicht, da die Schluffe als Grundwasserstauer dienen und an ihrer Oberkante zahlreiche Quellen austreten. Andere Pro- 
ben aus tieferen Teilen des Profils sind so mit groben Gesteinsfragmenten durchsetzt, dass sich mit dem Steckzylinder keine geeigneten Proben gewinnen ließen.

\subsection{Altersbestimmung der „Schieferkohle“}

Aufgrund der Geländesituation wurde zunächst von einer Verdichtung der Sedimente durch Eisüberfahrung ausgegangen. Somit schien ein Prähochwürm-glaziales Alter der „Schieferkohle" am wahrscheinlichsten. Da die Umgebung der Fundstelle heute bewaldet ist, größere Holzreste in der "Schieferkohle" aber völlig fehlen, war anfangs angenommen worden, dass es sich wohl um keine eindeutig warmzeitliche Bildung handeln könne. Angesichts ihrer Höhenlage von etwa $1140 \mathrm{~m}$ könnte die Fundstelle schon bei einer relativ bescheidenen Absenkung der "Schneegrenze“ in die Nähe der Baumgrenze geraten sein. Aufgrund dieser Überlegungen war ursprünglich mit einem Frühwürm-interstadialen Alter der „Schieferkohle“ gerechnet worden.

Um die angenommene Altersstellung zu überprüfen, wurde Probenmaterial der "Schieferkohle" an das Leibniz-Labor für Altersbestimmung und Isotopenforschung der Universität Kiel geschickt, um an dem Material eine konventionelle Radiokarbon-Datierung durchführen zu lassen. Die Messungen ergaben ein unkalibriertes ${ }^{14} \mathrm{C}$-Alter von $11930 \pm 80$ Jahren BP (Labornummer: KI-4859). Weitere Radiokarbon-Alter wurden mit der AMS-Methode am Van de Graaff Laboratorium der Universität Utrecht bestimmt. Danach hat der $4 \mathrm{~cm}$ mächtige Basis-Horizont ein ${ }^{14} \mathrm{C}$-Alter von $11950 \pm$ 60 Jahren BP (Labornummer: UtC Nr. 11355), für den Horizont unmittelbar unter den überlagernden Kiesen ergibt sich dagegen ein Alter von $11620 \pm 80$ Jahren BP (Labornummer: UtC Nr. 11354)(Abb. 3 links).

Aufgrund dieser ${ }^{14} \mathrm{C}$-Datierungen fällt die Entstehung des Torfes ins Spätglazial der Würmeis-

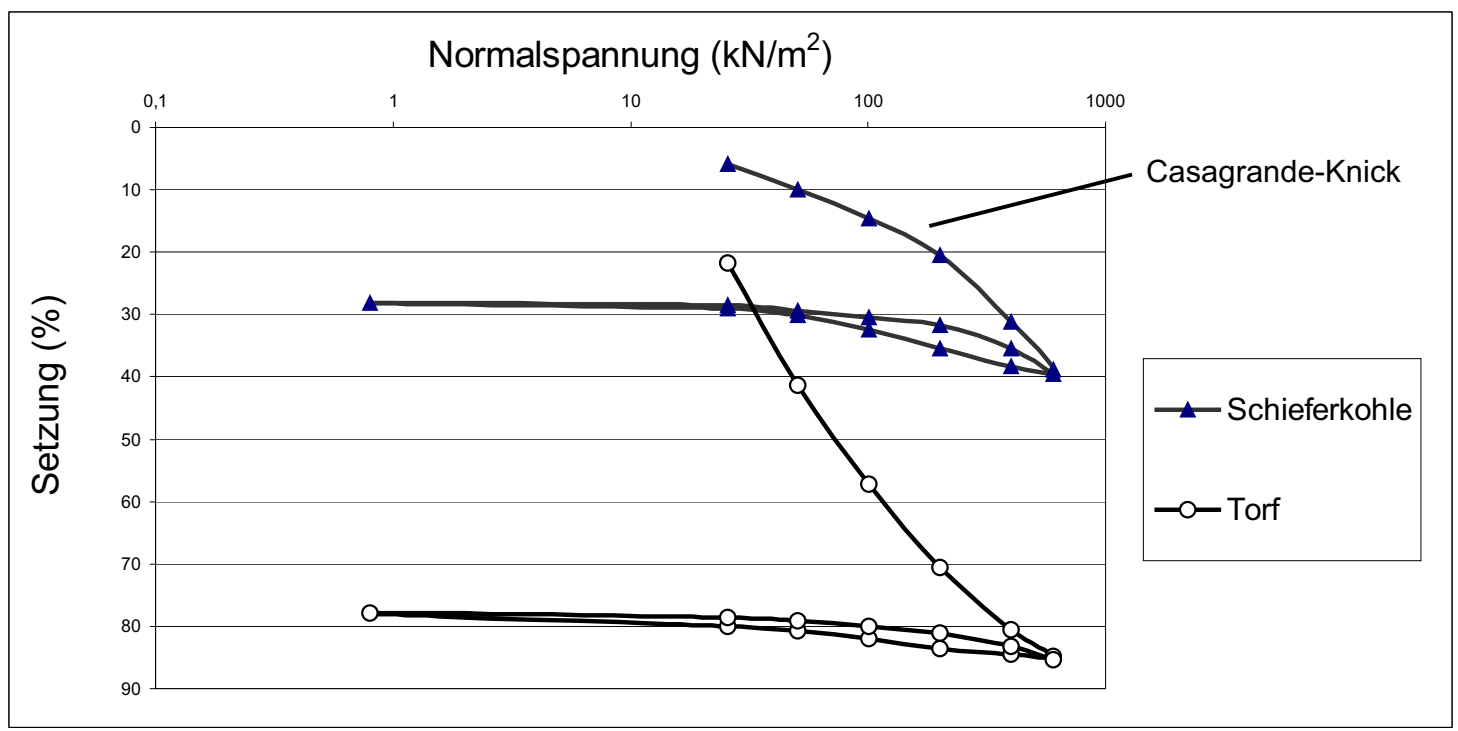

Abb. 4: Vergleich des Setzungsverhaltens der mutmaßlich eisüberfahrenen „Schieferkohle“ vom Sedererbach (oben) und eines nicht vorbelasteten Torfes aus einem Torfstich von Fürmoosen bei Kirchseeon im Alpenvorland (unten). Anhand des „Casagrande-Knickes“ in der Setzungskurve lässt sich die Größe der Vorbelastung des Materials abschätzen. 
zeit, genauer in den Beginn der spätglazialen Warmphase des Alleröd. Dafür sprechen auch die pollenanalytischen Untersuchungen durch Frau Dr. H. Schneider vom Geographischen Institut der Universität Jena. Vergleicht man die palynologischen Ergebnisse (Abschnitt 2) mit den Arbeiten von Kleinmann (1992, 1995) aus dem Ammersee und Ammann \& LotTer (1989) aus dem Rotsee, so lassen sich die untersuchten Pollenspektren gleichfalls dem Alleröd-Interstadial zuordnen, also der Pollenzone II nach Firbas (1949).

Nach den Paläotemperatur-Messungen, die Grafenstein et al. (1992: 150) an Ostrakodenschalen im Ammersee durchgeführt haben, herrschten zu dieser Zeit im Alpenvorland Jahresdurchschnittstemperaturen, die mit etwa $+6^{\circ} \mathrm{C}$ nur wenig unter den heutigen Temperaturen $\left(+8^{\circ} \mathrm{C}\right)$ liegen. Entsprechend dürften auch die Jahresmitteltemperaturen im Tal des Sedererbaches z.Z. der Bildung dieser "Schieferkohle" nicht all zu weit unter den heutigen gelegen haben. Die Pressung der „Schieferkohle" muss jünger sein und könnte mit einer möglichen Eisüberfahrung während der jüngeren Dryaszeit zusammenhängen. Der Eisvorstoß müsste folglich mit dem zentralalpinen EgesenStadium parallelisierbar sein (Patzelt \& BorTENSCHLAGER 1978). Damit könnte es sich um die jüngste bekannte „Schieferkohle“ Deutschlands handeln, für die eine Vorbelastung durch Gletschereis anzunehmen ist. Aufgrund ihres geringen Alters und ihrer Höhenlage sind Vergleiche mit anderen bekannten „Schieferkohlen" im nordalpinen Raum und im Alpenvorland nur schwer zu ziehen (z.B. BrunNACKeR i962, Heuberger i968, Keller \& Krayss 1997, Patzelt 1983, Stephan 1970). Einzig die "Schieferkohle“ von Imberg bei Sonthofen liegt ähnlich hoch, ist aber mit einem Radiokarbon-Alter von > 40000 Jahren deutlich älter (SCHWERD et al. 1983) und stammt angeblich aus einem Frühwürm-Interstadial.

\section{Der Sedererkar-Gletscher und die spätglaziale Depression der Schneegrenze}

Für eine denkbare Eisüberfahrung könnte der Vorstoß eines Lokalgletschers aus dem unmittelbar im Süden anschließenden Sedererkar verantwortlich sein. Die Eiszunge müsste, um das Gebiet mit der "Schieferkohle“ erreicht und $20 \mathrm{~m}$ hoch überdeckt haben zu können, um mindestens $700 \mathrm{~m}$ über den Karriegel und die Mittleren Sedererwände nach Norden hinaus vorgestoßen sein und dabei eine Höhendifferenz von mehr als $300 \mathrm{~m}$ überwunden haben.

Für die Entstehung eines Gletschers ist neben der nötigen Höhenlage und ausreichendem Niederschlag auch Exposition, Sonneneinstrahlung und die Möglichkeit der Akkumulation durch Lawinen zu berücksichtigen. Die Position des ideal ausgebildeten, amphitheaterförmigen Sedererkares ist für die Akkumulation großer Schneemengen sehr günstig. Es liegt an der extrem steilen Nordseite der Rindalphorn-Hochgrat-Kette, deren Kamm im Bereich des Sedererkares eine Höhe von ca. $1590 \mathrm{~m}$ erreicht. Der etwa $1450 \mathrm{~m}$ hoch liegende Boden des Sedererkares, in dem Erosionsreste von feinkörnigen Karsee-Sedimenten aufgeschlossen und erbohrbar sind (IrmLer 2001), wird von den übersteilten Oberen Sedererwänden überragt $(1570 \mathrm{~m})$, die aus steil nach Süden einfallenden Molassekonglomeraten aufgebaut werden. Dadurch wurde das Kar sicherlich nicht nur durch die an sich schon sehr hohen Niederschläge im Nordstau der Alpen, sondern auch durch Lawinen mit Schnee versorgt. Abgesehen von seiner Nordexposition und die dadurch bedingte geringe Sonneneinstrahlung wird das Kar im E vom 1737 m hohen Sedererstuiben und im W vom 1772 m hohen Buralpkopf flankiert, was eine zusätzliche Beschattung eines Kargletschers bedeutet.

Ein Kargletscher konnte sich während der Jüngeren Tundrenzeit im Sedererkar natürlich 


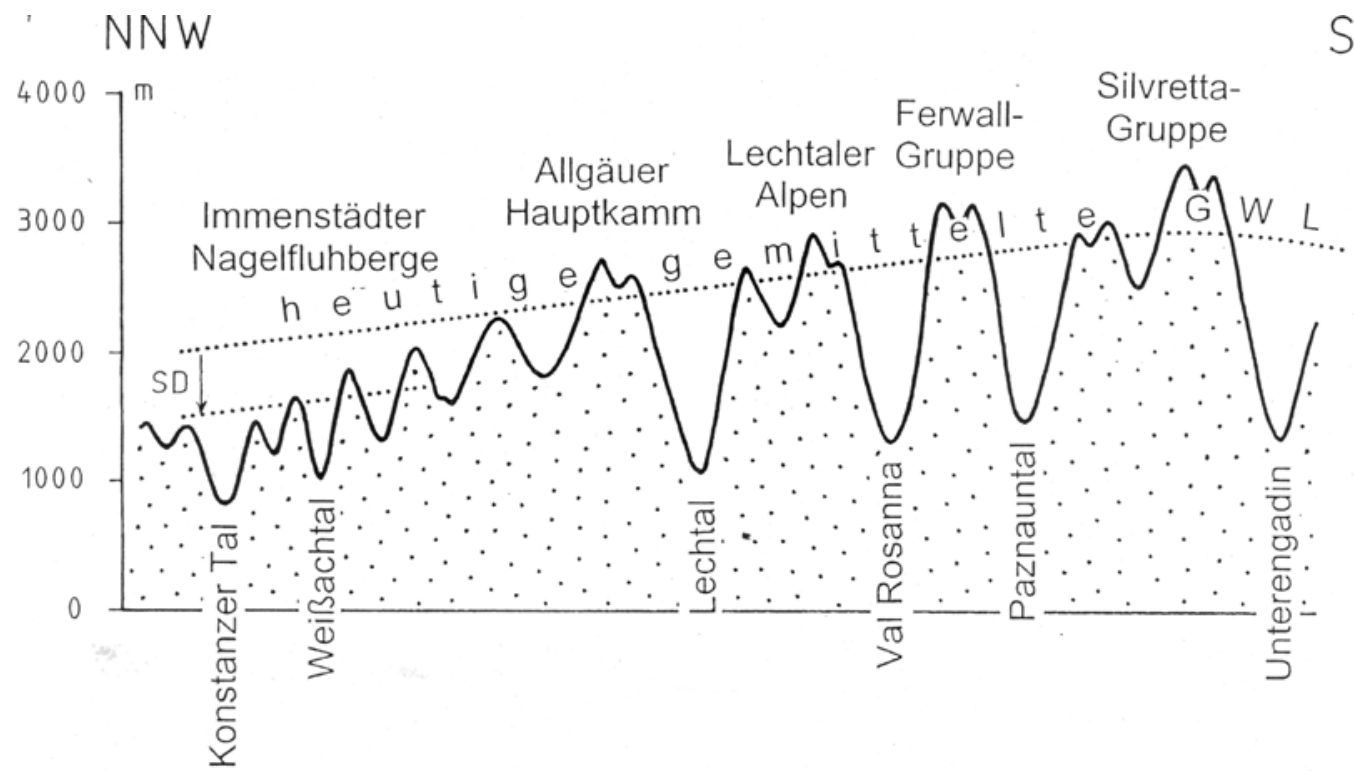

Abb. 5: Schematischer N-S-Schnitt durch die Ostalpen zwischen der Silvretta und dem Allgäu, mit Lage der heutigen Schneegrenze (GWL). Verwendet wurden u.a. die Angaben von Gross (1983), Vorndran (1970) sowie Schug \& Kunn (1993). Die hypothetische Verlängerung der gemittelten Höhenlage der Gleichgewichtslinie bis zum Alpennordrand dient der Rekonstruktion der spätglazialen Depression der Schneegrenze (SD) in den Immenstädter Nagelfluhbergen.

nur dann bilden, wenn die "Schneegrenze“ in dieser Zeit deutlich niedriger lag als heute. Die "Schneegrenze“ muss irgendwo zwischen dem Karboden $(1450 \mathrm{~m})$ und dem Sattel zwischen Sedererstuiben und Buralpkopf (1590 m) angenommen werden, also in einer Höhe von 1500 bis max. $1550 \mathrm{~m}$. Ist eine spätglaziale „Schneegrenzendepression" dieser Größenordnung überhaupt wahrscheinlich?

Die heutige "Schneegrenze" - sie wird in Anlehnung an Gross (1983) im folgenden als Mittel der Gleichgewichtslinie (GWL) bzw. „equilibrium line“ (EL) über mehrere Jahre verstanden - auf Gletschern liegt im Bereich der zentralen Ostalpen nach Gross (1983: 68) um die $3000 \mathrm{~m}$ hoch. Im Gebiet des Alpenhauptkammes liegt sie in der Silvrettagruppe, die sich genau südlich des Allgäus befindet, in einer Höhe von 2950 m (Abb. 5). Doch schon am Nordrand der Silvretta, bei Galtür, sinkt sie auf eine Höhe von nur mehr 2600 m ab (VorN-
DRAN 1970: 160 f.). In der noch weiter nördlich gelegenen Ferwallgruppe liegt die gemittelte Höhenlage der Gleichgewichtslinie nochmals deutlich niedriger. Für den im Wettersteingebirge gelegenen Höllentalferner (ENE-Expositon) - er befindet sich südöstlich des beschriebenen Schieferkohlevorkommens - nennt Hirtlreiter (1992) eine rezente Schneegrenze von 2375 m. Das nächstgelegene rezente Firnfeld ist der Schwarzmilzferner am Südhang der Mädelegabel im Bereich des Hauptkammes der Allgäuer Alpen. Nach Schug \& Kunn (1993) ist die "Schneegrenze" am Schwarzmilzferner bei $2450 \mathrm{~m}$ anzunehmen (Abb. 5). Im Gegensatz zu diesem Firnfeld ist das Sedererkar aber nordexponiert. Nach KuHN (1983) beträgt der Höhenunterschied zwischen den „Schneegrenzen" nord- und südexponierter Gletscher im Schnitt $172 \mathrm{~m}$ in den Nördlichen Kalkalpen. Folglich wäre die gemittelte Höhenlage der Gleichgewichtslinie im Gebiet der Mädelega- 
bel für einen (hier aber nicht vorhandenen) nordexponierten Gletscher bei knapp 2275 m anzunehmen. Interpoliert man die Abnahme der Gleichgewichtslinienhöhe von den Zentralalpen zum Alpenrand hin nach Norden, muss man eine hypothetische Lage der heutigen "Schneegrenze“ im Gebiet der Immenstädter Nagelfluhberge von $2100 \mathrm{~m}$ annehmen (Abb. 5). Es ist allerdings anzumerken, dass sie je nach Witterungsumständen bezüglich Form und Höhenlage von Jahr zu Jahr stark variieren kann (MaIsCh 1992).

Die klimatische Schwankung während der Jüngeren Dryaszeit hat in den Zentralalpen nach Patzelt (1983: 39) eine Absenkung der „Schneegrenze“ um bis zu 450 m bewirkt. Etwa vergleichbare Werte konnten von HiRTLReITER (1992) im Wettersteingebirge (Höllental) festgestellt werden. Er gibt für einen sicher bestimmten Moränenstand aus der Jüngeren Dryas eine Schneegrenzdepression von 395 m (Bezugsniveau 1990), für einen vermuteten Gletscherstand aus der Jüngeren Dryas oder älter eine Depression von $445 \mathrm{~m}$ (Bezugsniveau 1990) an. Geht man von der oben abgeleiteten Höhenlage der heutigen hypothetischen "Schneegrenze“ im Gebiet der RindalphornHochgrat-Kette aus, errechnet sich hier eine spätglazial-stadiale Schneegrenzhöhe von ca. $1650 \mathrm{~m}$. Sie liegt damit etwa $200 \mathrm{~m}$ über dem Niveau des heutigen Karbodens und etwa $100 \mathrm{~m}$ über der oben aufgrund von geomorphologischen Überlegungen ermittelten Höhenlage der Gleichgewichtslinie. Dieser unzulängliche Rekonstruktionsversuch würde also gegen die Möglichkeit der Entstehung eines Kargletschers an dieser Stelle sprechen. Allerdings werden einige günstige Lagefaktoren nicht berücksichtigt, die vielleicht dazu beitragen würden, die spätglaziale „Schneegrenze“ lokal um mehr als $100 \mathrm{~m}$ weiter nach unten zu drücken.

Ein anderer Lösungsansatz für die Ermittlung der gemittelten Höhenlage der Gleichgewichtslinie während der Jüngeren Dryaszeit (u.a. KeL-
LER \& Krayss 1997) besteht in der Koppelung der "Schneegrenze“ an die Jahresmitteltemperatur. So führt nach Keller \& Krayss (1997) eine Temperaturänderung von $0,4^{\circ} \mathrm{C}$ bis $0,6^{\circ} \mathrm{C}$ zum Ansteigen oder Absinken der „Schneegrenze" um jeweils $100 \mathrm{~m}$. Da der Unterschied der Jahresmitteltemperatur zwischen der jüngeren Dryaszeit und heute nach Grafenstein et al. (1992: 150) etwas mehr als $6^{\circ} \mathrm{C}$ betragen haben soll, errechnet sich eine hypothetische "Schneegrenze" von etwa $1400 \mathrm{~m}$ Höhe im Untersuchungsgebiet. Somit wäre eine späteiszeitliche Vergletscherung des $1450 \mathrm{~m}$ hoch gelegenen Sedererkares während des Egesen-Stadiums durchaus denkbar.

\section{Die jüngste Landschaftsgeschichte im oberen Weißachtal}

Versucht man all diese Beobachtungen und Erkenntnisse zu einem einheitlichen Bild zusammenzufügen, würde die Landschaftsgeschichte dieser Gegend in der ausgehenden Würmeiszeit in einem völlig neuen Licht erscheinen. Seit den grundlegenden Arbeiten von Patzelt \& Bortenschlager $(1969,1978)$ in den zentralen Ostalpen ist klar geworden, dass das Rückschmelzen der großen Vorlandgletscher bis zum Alpenrand in wesentlichen Teilen noch im ausgehenden Hochglazial erfolgte und die Gletscher sich noch während des Spätglazials in die Hochlagen der Alpen zurückgezogen haben müssen. Dadurch scheint gesichert, dass die meisten der deutlich ausgebildeten Wallsysteme, die die nordalpinen Kare umkränzen, größtenteils nicht aus dem frühen Holozän, wie man bis dahin vielfach angenommen hatte (z.B. Heuberger 1968), sondern aus dem Spätglazial stammen müssten. Niemand dürfte aber bisher damit gerechnet haben, dass die Klimadepression noch an der Wende vom Spät- zum Postglazial so kräftig war, dass sie in der Jün- 
geren Dryaszeit einen derart weiten Eisvorstoß (Egesen-Stadium) aus einem vergleichsweise ziemlich tief liegenden Kar auslösen konnte. Die Lokalgletscher an der übersteilten Nordflanke der Rindalphorn-Hochgrat-Kette sind während der Würmeiszeit wohl mehrfach vorgestoßen. Jedoch nur im Hochwürm dürften einige Gletscherzungen vorübergehend den Grund des Weißachtales erreicht haben. Das waren vor allem Gletscher, die Karen unter dem Hochgrat, dem Rindalphorn (Rindalpkar) und dem Buralpkopf (Sedererkar) entstammten. Doch nur die beiden östlichsten dieser Kargletscher, der Sedererkar- und der RindalpkarGletscher, konnten zeitweise echte Talgletscher ausbilden. Wie sich an sehr deutlichen und kaum verwitterten Moränenwällen rekonstruieren lässt, schob sich der aus dem Rindalpkar stammende Talgletscher etwa $2,5 \mathrm{~km}$ weit im Rindalptobel bis zu seiner Einmündung in die Weißach vor. Der Sedererkar-Gletscher schob sich mindestens $3 \mathrm{~km}$ weit über das Becken der Ehrenschwang-Alpe hinaus bis zu der Unteren-Eck-Alpe im Weißachtal vor. Die Gletscherstirn wird durch deutliche Moränenwälle nachgezeichnet, die etwa $400 \mathrm{~m}$ westlich der Unteren Eck-Alpe das Tal queren. Die Verbreitung glazigener Ablagerungen weist aber darauf hin, dass diese Gletscherzunge zeitweise wohl bis zur Einmündung des Rindalptobels nach W gereicht haben muss. Stillwassersedimente im Weißachtal deuten darauf hin, dass im Zungenbecken der Ehrenschwang-Alpe, aber auch östlich der Einmündung des Rindalpkar-Gletschers, zeitweise Schmelzwasserseen aufgestaut worden sind.

Im Spätglazial - zumindest im Alleröd - ist der Sedererkar-Gletscher in das $1450 \mathrm{~m}$ hoch gelegene Sedererkar zurückgeschmolzen und vielleicht vorübergehend völlig verschwunden. Das vom Eis unterhalb der Unteren Sedererwände zurückgelassene Material wurde wegen des starken Gefälles und des in den kälteren Abschnitten des Spätglazials herrschenden Frost- klimas gelifluidal umgelagert und mit den hier anstehenden Molassemergeln vermischt. Die im Talboden akkumulierten Fließerden bilden die Basis des oben beschriebenen Aufschlusses (Abb. 3). In flachen Depressionen des unruhigen Geländes entstanden in der Nähe des Sedererbaches kleine Tümpel und Flachmoore mit geringmächtigen Torfen. Bachhochwässer lagerten in diesen Mulden immer wieder sandig-schluffige Sedimente ab, wodurch die Torfbildung vorübergehend unterbrochen wurde. Bei einer erneuten Klimaverschlechterung in der Jüngeren Dryaszeit - die Jahresdurchschnittstemperatur sank selbst im Alpenvorland auf etwa $0^{\circ} \mathrm{C}$ ab - könnte es einen weiteren Vorstoß des Sedererkar-Gletschers gegeben haben. Die Eiszunge schob sich mindestens $700 \mathrm{~m}$ über den Karriegel hinaus, überwand eine Höhe von fast $300 \mathrm{~m}$ und stieß noch etwas über die Flachmoore am Sedererbach vor (Egesen-Stadium). Dabei könnten die Torfe überfahren und gepresst worden sein. Angesichts der rekonstruierbaren geringen Mächtigkeit des Eises von max. $22 \mathrm{~m}$ sollte der Gletscher damals nicht allzu weit nördlich von diesen Torfen gestirnt haben. Deutliche Moränenwälle sind hier allerdings nicht erhalten geblieben, könnten allerdings unter den weitläufigen Schwemmfächern südlich der Ehrenschwangalpe verborgen sein. Bei der fortschreitenden Klimaerwärmung an der Grenze vom Spät- zum Postglazial kam es zu einem neuerlichen raschen Rückschmelzen des Eises. Aus dieser Phase stammen vermutlich die Schmelzwasserkiese im Hangenden der "Schieferkohlen". Die Genese und der Erhalt der "Schieferkohle“. ist der geschützten Lage am Fuße der steilen Sedererwände zu verdanken. Ähnlich klare Belege für einen so jungen Eisvorstoß fehlen bisher jedoch in der Umgebung anderer Kare der Rindalphorn-Hochgrat-Kette (Irmler 200i, Rogozinski i 998, Vollmayer I958, Dongus I982,1983). 


\section{Danksagung}

Für die pollenanalytische Untersuchung des Aufschlusses bedanken wir uns bei Frau Dr. H. Schneider von der Friedrich-Schiller-Universität Jena. Unser Dank gilt auch Herrn Dr. Erlenkeuser vom Leibniz-Labor für Altersbestimmung und Isotopenforschung der Universität Kiel und Herrn Dr. K. van der Borg vom R. J. Van de Graaff Laboratorium der Universität Utrecht für die ${ }^{14} \mathrm{C}$-Datierungen. Für die Durchführung und Auswertung ergänzender Kompressionsversuche bedanken wir uns bei Frau Dipl.-Geol. Marion Nickmann und Herrn Matthias Bober. Zudem sind wir auch Herrn Dr. R. Plinninger vom Lehrstuhl für Allgemeine, Angewandte und Ingenieur-Geologie der TU München, Herrn Dr. M. Fiebig vom Bayerischen Geologischen Landesamt und Frau Dipl.-Geol. D. Frieling (Olching) für die kritische Durchsicht des Textes zu Dank verpflichtet.

\section{Literaturverzeichnis}

Ammann, B. \& Lotter, A.F. (1989): Late Glacial radiocarbon- and palynostratigraphie on the Swiss Plateau.- Boreas, 18: 109-126; Oslo.

BöLLING, W.H. (1971): Zusammendrückung und Scherfestigkeit von Böden. Anwendungsbeispiele und Aufgaben.- 174 S.; Wien (Springer).

BRUNNACKER, K. (1962): Das Schieferkohlenlager von Pfefferbichl bei Füssen.- Jber. U. Mitt. Oberrh. Geol. Ver., 44: 43-60; Stuttgart.

BüHRING， Ch. (1993): Erläuterungen zur geologischen Karte des Gebietes zwischen Oberstaufen und Missen im Allgäu (Faltenmolasse und Quartär).Diplomkartierung am Lehrstuhl für Geologie der TU München, unveröffentlicht: 78 S., 1 geol. Kt. 1: 10 000; Garching.

DIN 4021 (1990): Baugrund, Aufschluß durch Schürfe und Bohrungen.- Entwurf 10/90, 27 S.; Berlin, Wien, Zürich (Beuth).

DIN 18135 (1999): Eindimensionaler Kompressionsversuch, Baugrund, Untersuchungen von Bodenproben.- Entwurf 6/99, 38 S.; Berlin, Wien, Zürich (Beuth).

Dongus, H. (1982): Über die eiszeitliche Vergletscherung des westlichen Hochallgäus und die glaziale Überformung des Alpseeund des oberen Weißachtales.- Ber. z. dt. Landeskunde, 56: 219-238; Trier.

Dongus, H. (1983): Erläuterungen zur Geomorphologischen Karte 1: 25000 der Bundesrepublik Deutschland, GMK 25 Blatt 14 Nr. 8426 Oberstaufen.- 46 S., 1 geomorph. Kt.; Berlin.

Firbas, F. (1949): Spät- und nacheiszeitliche Waldgeschichte Mitteleuropas nördlich der Alpen. I. Allgemeine Waldgeschichte.- 480 S.; Jena (Fischer).

Grafenstein, U. von, Erlenkeuser, H., Müller, J. \& Kleinmann-Eisenmann, A. (1992): Oxygen Isotope Records of Benthic Ostracods in Bavarian Lake Sediments; Reconstruction of Late and Post Glacial Annual Air Temperatures.- Naturwissenschaften, 79: 145-152; Heidelberg.

Gross, G., Kerschner, H. \& Patzelt, G. (1976): Methodische Untersuchungen über die Schneegrenze in alpinen Gletschergebieten.- Zeitschrift für Gletscherkunde und Glazialgeologie, 12 (2): 223-251; Innsbruck.

Gross, G. (1983): Die Schneegrenze und die Altschneelinie in den österreichischen Alpen.- Innsbrucker Geographische Studien, 8: 59-76; Innsbruck.

Hirtlreiter, G. (1992): Spät- und postglaziale Gletscherschwankungen im Wettersteingebirge und seiner Umgebung.- Münchener Geographische Abhandlungen, B 15: 154 S.; München. 
Heuberger, H. (1968): Die Alpengletscher im Spät- und Postglazial, eine chronologische Übersicht.- Eiszeitalter und Gegenwart, 19: 270-275; Stuttgart.

IRMLER, R. (2001): Erläuterungen zur geologischen Karte des oberen Weißachtales südwestlich von Immenstadt im Allgäu (Faltenmolasse, Quartär) mit speziellen Untersuchungen zur Ursache von Hangbewegungen.- Diplomarbeit und Diplomkartierung am Lehrstuhl für Geologie der TU München, unveröffentlicht: 77 S.; 1 geol. Kt. 1: 10 000; München.

Jä́кLI, H. (1970): Die Schweiz zur letzten Eiszeit.- In: Atlas der Schweiz, Blatt 6, 1Kt. 1: 500 000; Wabern-Bern (Eidgen. Landestopographie).

Keller, O. \& Krayss, E. (1997): Eiszeit, Relief und Moorstandorte.- Ber. St. Gall. Natw. Ges., 88: 33-54; St. Gallen.

Kleinmann, A. (1992): Nachweis spät- und postglazialer Seespiegelschwankungen am Ammersee mittels pollenanalytischer und sedimentologischer Untersuchungen.- Diss. TU München: 139 S.; Garching.

KLeinmann, A. (I995): Seespiegelschwankungen am Ammersee: ein Beitrag zur spät- und postglazialen Klimageschichte Bayerns.-Geologica Bavarica, 99: 253-367; München.

Kunn, M. (1983): Die Höhe der Schneegrenze in Tirol, berechnet aus Fliris klimatischen Profilen.- Innsbrucker Geographische Studien, 8: 85-91; Innsbruck.

Maisch, M. (1992): Die Gletscher Graubündens. Rekonstruktion und Auswertung der Gletscher und deren Veränderungen seit dem Hochstand von 1850 im Gebiet der östlichen Schweizer Alpen (Bündnerland und angrenzende Regionen). Teil A (324 S.): Grundlagen-Analysen-Ergebnisse. Teil B (124 S.): Verzeichnisse-Datenlage-Gletscherkarten. Physische Geographie, Vol. 33; Univ. Zürich.

PAST, B.A. (2001): Erläuterungen zur geologi- schen Karte des Gebietes südwestlich des Alpsees bei Immenstadt im Allgäu (Faltenmolasse, Quartär), mit einer speziellen, hydrogeologischen Darstellung des Landkreises Lindau.- Diplomarbeit und Diplomkartierung am Lehrstuhl für Geologie der TU München, unveröffentlicht: 125 S., 1 geol. Kt. 1: 10 000; München.

Patzelt, G. (1983): Die spätglazialen Gletscherstände im Bereich des Mieslkopfes und im Arztal, Tuxer Voralpen, Tirol.- Innsbrucker Geographische Studien, 8: 35-44; Innsbruck.

Patzelt, G. \& Bortenschlager, S. (1969): Warmzeitliche Klima- und Gletscherschwankungen im Pollenprofil eines hochgelegenen Moores (2270 m) der VenedigerGruppe.- Eiszeitalter und Gegenwart, 20: 116-122; Öhingen.

Patzelt, G. \& Bortenschlager, S. (1978): Zur Chronologie des Spät- und Postglazials im Ötztal und Inntal (Ostalpen, Tirol).- In: Frenzel, B. [Hrsg.] (1978), Führer zur Exkursionstagung des IGCP-Projektes 73/1/ 24 „Quaternary Glaciation in the Northern Hemisphere", vom 5. Bis 13. September 1976: 185-197; Bonn - Bad Godesberg.

Prinz, H. (1997): Abriß der Ingenieurgeologie.- 3. Aufl., 546 S.; Stuttgart (Enke).

RogozinsKi, Th. (1998): Erläuterungen zur Geologischen Karte des Gebietes zwischen Oberstaufen und dem Hochgrat im Allgäu (Faltenmolasse, Quartär), mit speziellen Untersuchungen an fossilen Karseen der Hochgratkette.- Diplomarbeit und Diplomkartierung am Lehrstuhl für Geologie der TU München, unveröffentlicht: 106 S., 1 geol. Kt. 1: 10 000; Garching.

Sailer, R. \& Kerschner, H. (1999): Equilibrium-line altitudes and rock glaciers during the Younger Dryas cooling event, Ferwall group, western Tyrol, Austria. Annals of Glaciology, 28: 141-145; Cambridge.

Scholz, H. (1995): Bau und Werden der 
Allgäuer Landschaft.- 305 S.; Stuttgart (Schweizerbart).

Schug, J. \& Kunn, M. (1993): Der Schwarzmilzferner in den Allgäuer Alpen: Massenbilanz und klimatische Bedingungen.- Zeitschrift für Gletscherkunde und Glaziologie, 29 (1): 55-74; Innsbruck.

Schwerd, K., Ebel, R. \& Jerz, H. (1983): Geologische Karte von Bayern 1: 25 000, Erläuterungen zum Blatt Nr. 8427 Immenstadt.- 258 S., 1 geol. Kt.; München (Bayer. Geol. L.-Amt).

Stephan, W. (1970): Frühwürmzeitliche Schieferkohlevorkommen im Bereich des östlichen Isar-Vorlandgletschers.- Geologica Bavarica, 63: 217-230; München.

Vollmayr, Th. (1958): Geologische Karte von Bayern 1: 25 000, Erläuterungen zum Blatt Nr. 8426 Oberstaufen.- 41 S., 1 geol. Kt.; München (Bayer. Geol. L.-Amt).
Vorndran, G. (1970): Die Höhe der Schneegrenze in der Silvrettagruppe.- Mitt. Geogr. Ges. München, 55: 155-168; München.

Weinhardt, R. (1973): Rekonstruktion des Eisstromnetzes der Ostalpennordseite zur Zeit des Würmmaximums mit einer Berechnung seiner Flächen und Volumina.- Heidelberger Geogr. Arb., 38: 158-178, 1 Kt. 1: 1000 000; Heidelberg.

Wimmer, A. (1998): Erläuterungen zur geologischen Karte des Gebietes südwestlich von Oberstaufen im Allgäu (Faltenmolasse, Quartär), mit besonderen Untersuchungen zur Stratigraphie der Unteren Süßwassermolasse und des Quartärs.- Diplomarbeit und Diplomkartierung am Lehrstuhl für Geologie der TU München, unveröffentlicht: 189 S., 1 geol. Kt. 1: 10 000; Garching. 